\title{
Multimodal imaging of refractory Candida chorioretinitis progressing to endogenous endophthalmitis
}

\author{
Jeremy A Lavine and Mihai Mititelu*
}

\begin{abstract}
Background: Endogenous fungal endophthalmitis is a serious vision-threatening condition that occurs in immunosuppressed patients with candidemia.

Findings: We report a complicated case of Candida albicans chorioretinitis that progressed to endophthalmitis. The patient required intravitreal and systemic anti-fungal medications with pars plana vitrectomy for successful treatment. Multimodal imaging using fundus photography, fluorescein angiography, spectral domain optical coherence tomography, and fundus autofluorescence was obtained throughout treatment. These modalities localized the Candida infection in the choroid, penetrating Bruch's membrane, the retinal pigment epithelium, and the retina to enter the vitreous cavity. This infectious route resulted in loss of the retinal pigment epithelium, photoreceptors, and outer retinal layers, with scar formation that resulted in vision loss and increased future risk of choroidal neovascular membranes.
\end{abstract}

Conclusions: Multimodal imaging of C. albicans chorioretinitis allows for accurate diagnosis, assessment of response to therapy, and prognosis for visual recovery and future complications.

Keywords: Endophthalmitis; Chorioretinitis; Candida albicans; Optical coherence tomography; Fundus autofluorescence; Fluorescein angiography

\section{Findings}

\section{Introduction}

Endogenous fungal endophthalmitis is a serious visionthreatening condition. In the United States, yeasts are the most common causative organism, accounting for $75 \%$ of cases [1]. Among yeasts, Candida albicans is the most common pathogen [1]. In $14 \%$ of patients with Candidemia, ocular complications occur [2]. The majority of these patients develop chorioretinitis with $1.6 \%$ of patients advancing to endophthalmitis [2]. In patients with yeast endophthalmitis, visual acuity outcomes can be poor with only $56 \%$ of eyes achieving vision of $20 / 200$ or better [1].

This case report describes the clinical course of a patient with $C$. albicans chorioretinitis that progressed to recalcitrant endophthalmitis. Using multimodal imaging, we chronicle the features of chorioretinitis through multiple medical and surgical therapies.

\footnotetext{
* Correspondence: mititelu@wisc.edu

Department of Ophthalmology and Visual Sciences, University of

Wisconsin-Madison, 2870 University Avenue, Room 206, Madison, WI 53705, USA
}

\section{记 Springer}

(c) 2015 Lavine and Mititelu. Open Access This article is distributed under the terms of the Creative Commons Attribution 4.0 International License (http://creativecommons.org/licenses/by/4.0), which permits unrestricted use distribution, and reproduction in any medium, provided you give appropriate credit to the original author(s) and the source, provide a link to the Creative Commons license, and indicate if changes were made. The Creative Commons Public Domain Dedication waiver (http://creativecommons.org/publicdomain/zero/1.0/) applies to the data made available in this article, unless otherwise stated.

\section{Case Report}

A 48-year-old male presented with blurred central vision in his right eye. Past ocular history included bilateral pseudophakia. Medical history was significant for a 4-year history of rheumatoid arthritis on oral prednisone $15 \mathrm{mg}$ daily and a recent history of nephrolithiasis. One month prior to presentation, his nephrolithiasis was treated with extracorporeal shock wave lithotripsy and ureteral stent placement. One week after lithotripsy, urine cultures grew C. albicans and the patient started oral fluconazole $300 \mathrm{mg}$ daily. For 3 weeks, the patient complained of blurred central vision in the right eye without redness or floaters.

On examination, visual acuity was 20/100 OD and 20/ 20 OS. Pupils, intraocular pressures, and visual fields were normal. The anterior segment showed bilateral pseudophakia with no inflammation. Posterior segment exam demonstrated clear media without vitritis and a white, elevated foveal infiltrate (half disk diameter) with indiscrete borders (Fig. 1a). Fluorescein angiography (FA) displayed an early hyperfluorescent lesion with late staining 

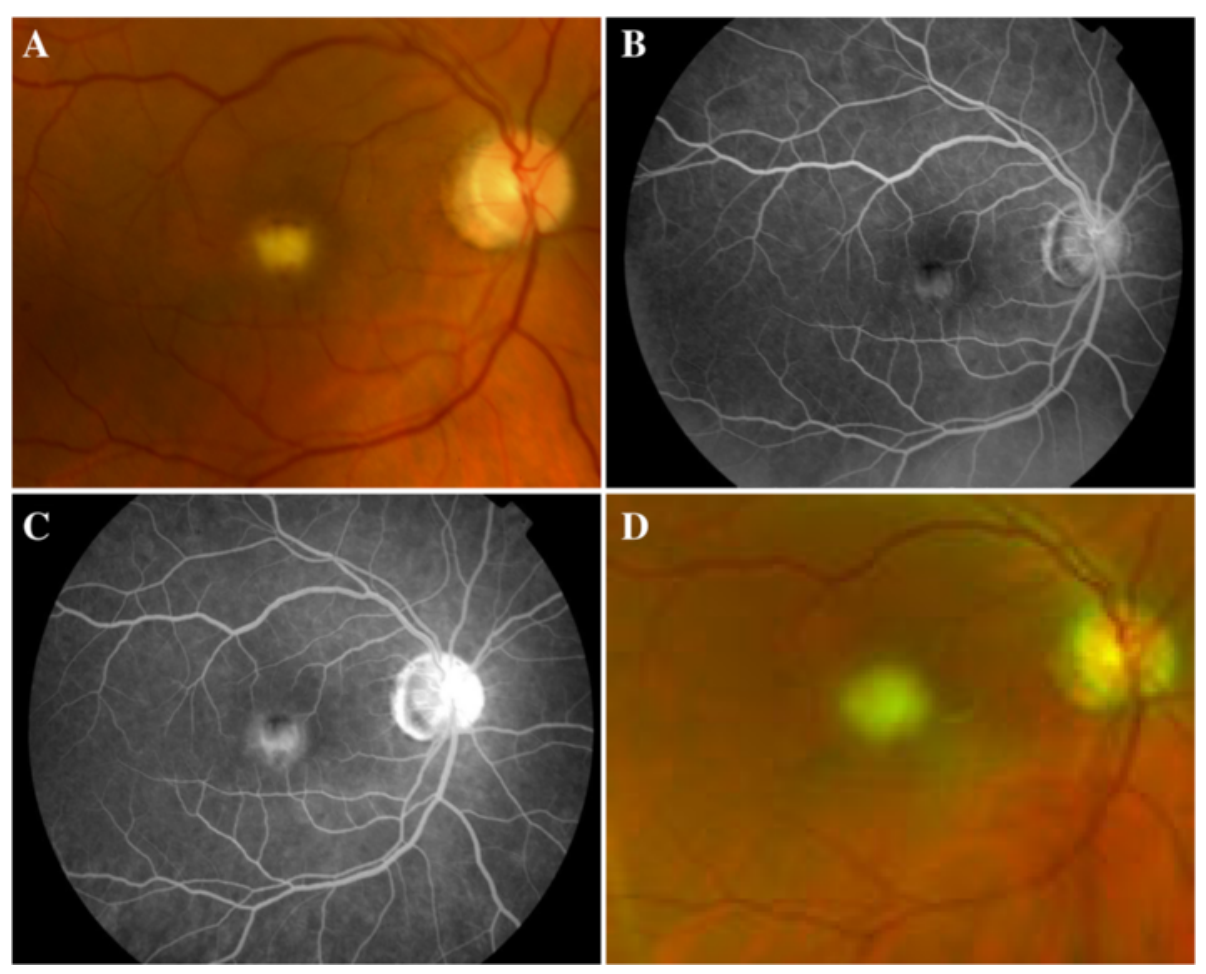

Fig. 1 Initial appearance of the chorioretinitis. Color fundus photograph (a) showing the white, elevated, and fluffy chorioretinal lesion. Early (b) and late (c) phases of the FA demonstrating early hyperfluorescence and late staining. Color fundus photography 2 weeks later displaying significant vitritis that obscures retinal detail (d)

(Fig. 1b, c). In the context of his Candiduria, a diagnosis of Candida chorioretinitis was made and his fluconazole was increased to $600 \mathrm{mg} /$ day. The following day, the ureteral stent was removed without catheterization and blood cultures grew C. albicans. Although an infectious disease consult was requested on the day of presentation, the patient was seen 1 week later, and the fluconazole dose was increased to $800 \mathrm{mg}$ daily.

The patient was lost to follow-up and presented 2 weeks later with new floaters OD. On examination, visual acuity remained 20/100 OD, the anterior segment was unchanged, and the posterior segment displayed vitritis with a fluffy chorioretinal lesion (Fig. 1d). Candida endophthalmitis was diagnosed, the patient received a same-day intravitreal injection of amphotericin B $(5 \mu \mathrm{g} / 0.1 \mathrm{~mL})$ and was placed on intravenous amphotericin $B(0.1 \mathrm{mg} / \mathrm{mL})$. The patient developed acute kidney injury (AKI) 1 week after initiation of treatment, requiring discontinuation of amphotericin B and resumption of fluconazole. At this time, blood cultures were negative for $C$. albicans.

One week after intravitreal amphotericin $B$, there was improvement in the fluffy appearance of the macular lesion. Spectral domain optical coherence tomography (SD-OCT) demonstrated an elevated, hyperreflective foveal lesion at the vitreoretinal inferface that appeared to be emanating from the retinal pigment epithelium (RPE), with obscuration of the retinal layers (Fig. 2a). The patient received three intravitreal voriconazole injections $(100 \mu \mathrm{g} / 0.1 \mathrm{~mL})$ and was placed on systemic voriconazole ( $200 \mathrm{mg}$ twice daily) therapy. After 2 months, the lesion flattened and developed welldemarcated borders but vitritis persisted. SD-OCT displayed an improving but still elevated hyperreflective lesion now localized at the nerve fiber layer with persistent focal discontinuity of the RPE (Fig. 2b). At its borders, the inner retinal architecture showed improved definition, but the outer retinal layers displayed atrophy with increased transmission defect. Due to the persistent vitreous infiltrates, the patient underwent pars plana vitrectomy (PPV). The postsurgical SD-OCT showed continued regression of the elevated, hyperreflective lesion (Fig. 2c). The borders demonstrated preserved inner retinal layers with atrophy of the outer retina and choroidal hyperreflectivity from transmission defect. The patient continued systemic voriconazole for an additional month without further intravitreal therapy.

Final multimodal imaging displayed a foveal scar (Fig. 3a). On fundus autofluorescence (FAF), the scar is hypofluorescent consistent with RPE loss (Fig. 3b). On SDOCT, the outer retina is atrophic, the RPE layer is absent, a 

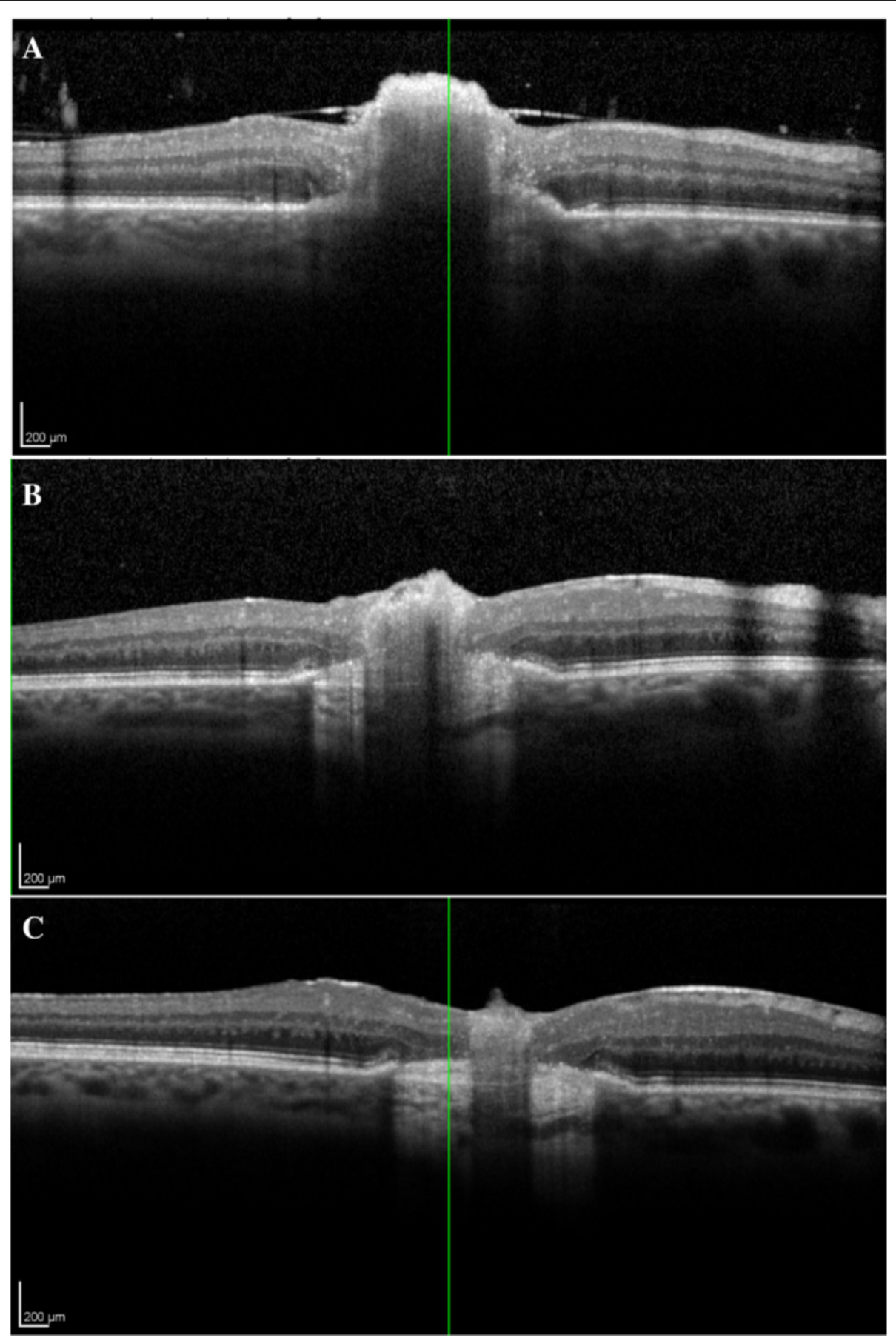

Fig. 2 Regression of the chorioretinal lesion. SD-OCT imaging performed 1 week after intravitreal amphotericin B treatment (a), 2 months after three intravitreal voriconazole injections (b), and after PPV (c). The inner retinal hyperreflective lesion regresses with time. At its borders, the RPE is elevated and discontinuous with loss of outer plexiform, outer nuclear, external limiting membrane, IS/OS, and RPE. Inner retinal tissues are preserved and subretinal scar is present

subretinal scar underlies the inner retinal layers, and a postinflammatory epiretinal membrane (ERM) co-exists (Fig. 3c). The photoreceptor loss and RPE atrophy primarily caused a final visual acuity of 20/100 OD.

\section{Discussion}

We present a complicated case of $C$. albicans chorioretinitis that progressed to endogenous endophthalmitis, requiring multiple local and systemic antifungal medications and 


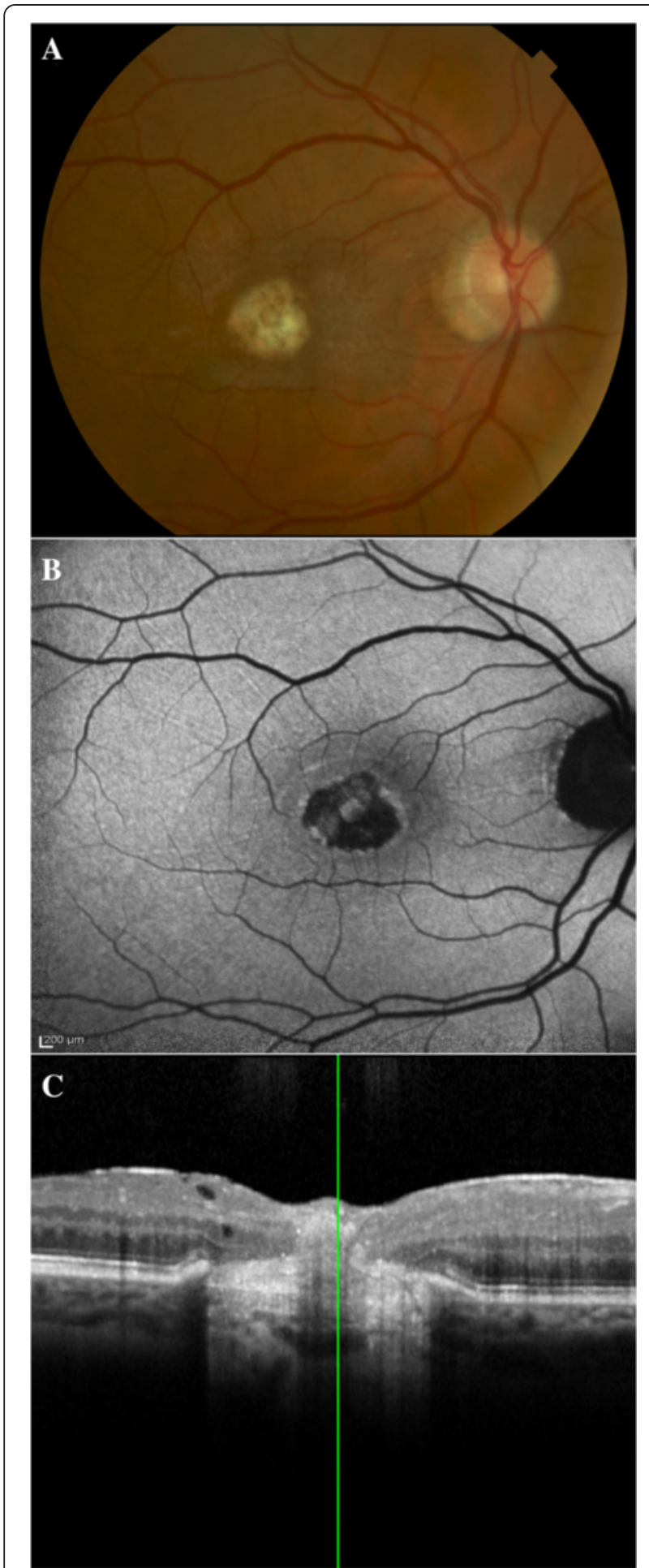

Fig. 3 Final appearance of the chorioretinal lesion. Color fundus photography (a), FAF (b), and SD-OCT (c) were performed 2 months after PPV. A subfoveal scar is present (a) with destruction of outer plexiform, outer nuclear, external limiting membrane, IS/OS, and RPE (c). The inner retinal layers, however, are preserved (c). The FAF shows loss of autofluorsescence corresponding to RPE destruction (b)
PPV. Risk factors for endogenous yeast endophthalmitis include hospitalization, surgery, cancer, diabetes, intravenous drug use, and indwelling catheters [1]. Risk factors for this particular case include recent lithotripsy of renal calculi and immunosuppression from prednisone therapy [3]. It is common for lithotripsy to cause asymptomatic Candiduria; in this immunocompromised patient, the Candiduria likely caused Candidemia, leading to chorioretinitis. In a prospective, multicenter study of 11 patients with chorioretinitis, none progressed to endophthalmitis [4]. Although limited by a small number of patients, this study suggests that our patient had been initially underdosed with fluconazole $300 \mathrm{mg}$ daily. Once Candida endophthalmitis developed, our patient received both intravitreal and systemic amphotericin B. However, therapy was changed to oral and intravitreal voriconazole after AKI from amphotericin B. Voriconazole is effective against fluconazole-resistant $C$. albicans strains and has excellent ocular penetration [5]. It has been shown that Candida species preferentially infect the vitreous and form loculated microabscesses, which may ultimately require vitrectomy for clearance [6]. In the case of our patient, despite stabilization of the infection with aggressive systemic and intravitreal antifungal management, PPV was required to decrease the fungal load.

To our knowledge, this is the first report using FA, SDOCT, and FAF to follow a case of Candida chorioretinitis progressing to endophthalmitis. Our patient initially presented with a white, elevated chorioretinal lesion (Fig. 1a). FA displayed a foveal lesion with early hyperfluorescence and late staining (Fig. 1b, c), consistent with prior reports of Candida chorioretinitis [7]. The lack of late leakage rules out choroidal neovascularization (CNV). However, the presence of vascular leakage near the lesion does not exclude Candida chorioretinitis as the cause of the macular lesion, as this angiogram pattern has been documented [8].

SD-OCT and FAF imaging help the clinician determine the route of infectious seeding, the etiology and treatment options, and the prognosis for visual recovery. SD-OCT findings early in the course of treatment demonstrated an elevated, hyperreflective lesion at the retina-vitreous interface with poorly defined borders and obscured underlying retinal detail (Fig. 2a). At the edge of the lesion, elevation of the RPE suggests that the lesion originated in the choroid. We suspect that this lesion is a focus of inflammatory and infectious material that locally infiltrated the macula. As the lesion was treated, the inner retinal hyperreflective lesion regressed but the borders demonstrated a persistently elevated, discontinuous RPE (Fig. 2b, c). These characteristics suggest that the Candida infection progressed via choroidal infiltration through Bruch's membrane and RPE, into the retina and the vitreous. We hypothesize that this seeding is secondary to spread through the short posterior ciliary artery rather than through the central retinal artery $[9,10]$ because of the 
initial presentation as an indolent chorioretinitis instead of an explosive endophthalmitis. Cho et al. previously evaluated Candida chorioretinitis with SD-OCT, demonstrating RPE elevation and outer retinal destruction in early, active lesions and inner retinal hyperreflective elevation with blockage in late, inactive lesions [11]. Our lesion showed SD-OCT characteristics of both early, active and late, inactive chorioretinitis stages as it evolved during the clinical course. We hypothesize that clinically and through multimodal imaging, our lesion demonstrated active features, especially given its pronounced hyperfluorescence on FA and its regression with treatment.

Vision loss in our patient occurred primarily due to photoreceptor loss and scarring. SD-OCT showed loss of outer plexiform, outer nuclear, external limiting membrane, and inner segment/outer segment (IS/OS) layers, and an area of thickened and hyperreflective subretinal scar tissue (Fig. 3c). FAF displayed lack of fluorescence centrally, indicating RPE destruction and confirming the presence of scar tissue (Fig. 3b). Not including the ring of hypofluorescent peripapillary atrophy, the scar is one disk diameter, which is twice the initial infiltrate. Thus, our imaging demonstrates centrifugal scar expansion and photoreceptor loss as the primary causes for vision loss. In other reports, vision loss occurred in the presence of macular edema [12,13], a clinical finding absent in this case.

Our patient remains at risk for further vision loss from potential CNV. FAF and SD-OCT imaging (Fig. 3) showed inflammatory and infectious destruction of Bruch's membrane and RPE destruction, creating a locus for potential development of $\mathrm{CNV}$. We suspect that once the integrity of the RPE layer has been violated, there is endophytic spread of the infection into the retina and vitreous, which increases the risk of further visual loss and the chance of requiring surgery. We emphasize that both SD-OCT and FAF highlight findings that were only previously demonstrable on histopathology. More importantly, they serve as non-invasive and readily available tools for monitoring progression and for informing the clinician regarding the effectiveness of various treatment modalities and prognosis for visual recovery.

\footnotetext{
Abbreviations

AKI: acute kidney injury; CNV: choroidal neovascular membrane; ERM: epi-retinal membrane; FA: fluorescein angiography; FAF: fundus autofluorescence; IS/ OS: inner segment/outer segment; OD: right eye; OS: left eye; OU: both eyes; PPV: pars plana vitrectomy; RPE: retinal pigment epithelium; SD-OCT: spectral domain optical coherence tomography.
}

\section{Competing interests}

The authors declare that they have no competing interests.

\section{Author's contributions}

$J$ was involved in the care of the above patient and is the primary author of the manuscript. MM was the supervising attending physician throughout the entire duration of the above patient's care. MM was the primary decision maker regarding the ordering and interpretation of tests, delivery of treatments, and overall care for the patient. Both authors read and approved the final manuscript.

\section{Author's information}

$J$ is a resident of ophthalmology at the University of Wisconsin-Madison. MM is an assistant professor of ophthalmology and medical retina specialist at the University of Wisconsin-Madison.

\section{Acknowledgements}

Neither author has received any grant support or research funding for this project.

Received: 16 February 2015 Accepted: 28 July 2015

Published online: 08 August 2015

\section{References}

1. Lingappan A, Wykoff CC, Albini TA et al (2012) Endogenous fungal endophthalmitis: causative organisms, management strategies, and visual acuity outcomes. Am J Ophthalmol 153:162-166.e1, doi: 10.1016/ j.ajo.2011.06.020

2. Oude Lashof AML, Rothova A, Sobel JD et al (2011) Ocular manifestations of candidemia. Clin Infect Dis 53:262-268. doi:10.1093/cid/cir355

3. Greenwald BD, Tunkel AR, Morgan KM et al (1992) Candidal endophthalmitis after lithotripsy of renal calculi. South Med J 85:773-774

4. Donahue SP, Greven CM, Zuravleff JJ, Eller AW (1994) Intraocular candidiasis in patients with candidemia: clinical implications derived from a prospective multicenter study. Ophthalmology 101(7):1302-1309

5. Riddell J, Comer GM, Kauffman CA (2011) Treatment of endogenous fungal endophthalmitis: focus on new antifungal agents. Clin Infect Dis 52:648-653. doi:10.1093/cid/ciq204

6. Rao NA, Hidayat AA (2001) Endogenous mycotic endophthalmitis: variations in clinical and histopathologic changes in candidiasis compared with Aspergillosis. Am J Ophthalmol 132:244-251

7. Vianna RNG, Filho JPS, Deschênes J, Burnier MN Jr (2005) Bilateral Candida chorioretinitis: involvement of the second eye after 3 years. Can J Ophthalmol 40:75-78. doi:10.1016/S0008-4182(05)80122-X

8. Alexandridou A, Reginald AY, Stavrou P, Kirkby GR (2002) Candida endophthalmitis after tattooing in an asplenic patient. Arch Ophthalmol 120:518-519

9. Griffin JR, Pettit TH, Fishman LS, Foos RY (1973) Blood-borne Candida endophthalmitis. Arch Ophthalmol 89:450-456. doi:10.1001/ archopht.1973.01000040452002

10. Kawanishi Y, Morinobu T, Shirakawa K, Ueno Y (1987) Histopathological studies of endogenous fungal endophthalmitis. Folia Ophthalmol Jpn 38:204-211

11. Cho M, Khanifar AA, Chan RVP (2011) Spectral-domain optical coherence tomography of endogenous fungal endophthalmitis. Retin Cases Brief Rep 5:136-140. doi:10.1097/ICB.0b013e3181cc2146

12. Chang Y, Yang CS, Lee FL, Lee SM (2012) Voriconazole for Candida endophthalmitis. OPHTHA 119:2414-2415, e4. doi: 10.1016/ j.ophtha.2012.06.020

13. Chavan R, Mustafa MZ, Narendran N et al (2012) A case of Candida albicans endophthalmitis with no predisposing risk factors and a distant source of infection. Case Rep Ophthalmol 3:277-282. doi:10.1159/000342135

\section{Submit your manuscript to a SpringerOpen ${ }^{\circ}$ journal and benefit from:}

- Convenient online submission

- Rigorous peer review

- Immediate publication on acceptance

- Open access: articles freely available online

- High visibility within the field

- Retaining the copyright to your article

Submit your next manuscript at $>$ springeropen.com 